\title{
The accuracy of post-traumatic orbital volume correction using pre- adapted titanium mesh (randomized controlled clinical prospective study)
}

\section{Original Article}

\author{
Eldek. $Y^{1}$, Sleem.H $H^{2}$ Katamesh.M $M^{2}$ Hasanin.F ${ }^{3}$ \\ ${ }^{1}$ Oral and Maxillofacial specialist, Nasser Institute Hospital, Cairo. \\ ${ }^{2}$ Oral and Maxillofacial Surgery, Faculty of Dentistry, Ain Shams University. \\ ${ }^{3}$ Oral and Maxillofacial Surgery, Faculty of Dentistry, Cairo University, Head of Oral \\ and Maxillofacial Surgery Department, Nasser Institute Hospital, Cairo.
}

\begin{abstract}
Aim: Is to evaluate the accuracy of orbital volume correction using pre-adapted titanium mesh with the aid of stl model of corrected orbit.

Patients and methods: Ten patients with unilateral orbital floor fracture were included. The cases were divided into two equal groups. Manual group where titanium mesh was manually adapted intra-operatively. STL group where titanium mesh was adapted pre-operatively using stl model of corrected orbit. Orbital volume measurements based on CT images were used to trace the degree of correction in each group. Paired samples $t$ test was used for statistical analysis.

Results: There was statistical significant difference in the correction of orbital volume in each group. However, comparison between the two groups revealed no statistical significant difference in the percentage of correction, where the mean percentage of correction in stl group was (102.8 \pm 4.4$)$ versus $(98.4 \pm 2.6)$ in the manual group.

Conclusion: Application of pre-adapted titanium mesh by the aid of stl model provide successful tool for correction of orbital volume secondary to orbital floor fracture with less operative time and effort especially in comminuted fractures.
\end{abstract}

Key Words: Adaptation, mesh, orbit, titanium, volume.

Received: 28 September 2018, Accepted: 26 November 2018

Corresponding Author: Sleem, Heba Abdulwahed, Department of Oral and Maxillofacial Surgery Ain Shams University, Cairo, Egypt, E-mail: drsleemh@gmail.com

ISSN: 2090-097X, October 2018, Vol. 9, No. 4

\section{INTRODUCTION}

Orbital fractures constitute about forty percent of head and neck trauma cases ${ }^{[1]}$. Floor and medial walls are most commonly involved with resultant increase in orbital volume $^{[2]}$. Clinical manifestation of increased orbital volume includes enophthalmos, vertical dystopia, diplopia, and limited ocular motility ${ }^{[3]}$. Many surgical techniques have been adopted for correction of orbital volume. Topography of the orbital floor (S shape in sagittal plane) is one of difficulty factors during insertion of reconstructive material. However there is a continuous search for the best method to achieve accuracy, feasibility, and reliability for restoration of the orbit ${ }^{[4]}$. There are a lot of reconstructive materials but titanium mesh implant is the most commonly used one $\mathrm{e}^{[5]}$. Intra-operative adaptation of titanium mesh after exposure and reduction of fractured bones is the traditional technique. However, this necessitates more dissection, multiple trials, and longer operative time especially in comminuted fracture. Computer aided surgical techniques allow virtual correction of fractured bones using the intact one as a reference with subsequent printing to achieve stl model of reduced orbit. The present study aimed to explore the accuracy of orbital volume correction using pre-adapted titanium mesh by stl model versus traditional technique in cases of unilateral orbital floor fracture.

\section{Methodology}

Ten adult male patients with unilateral orbital floor fracture were included in this study. Cases collected from the department of Oral and Maxillofacial Surgery, Faculty of Dentistry, Ain Shams University and Nasser institute hospital, Cairo.

Thin section CT scans in the axial, coronal, and sagital views with three dimensional reconstructions were done for definitive diagnosis and treatment planning (Fig.1). 

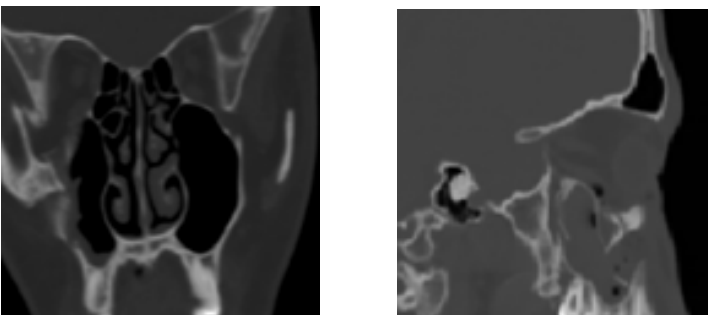

Fig.1: Preoperative CT showing disruption of the Rt orbital floor in coronal view (a) and sagittal view (b)

Detailed ophthalmic assessment was done for all patients to document associated ocular injuries. the cases were divided into two equal groups: stl group where titanium mesh was adapted pre-operatively on stl model and manual group where titanium mesh was adapted and fitted intra-operatively. Clinical photographs of both fractured and intact orbits were used for documentation of esthetic outcome (Fig.2).
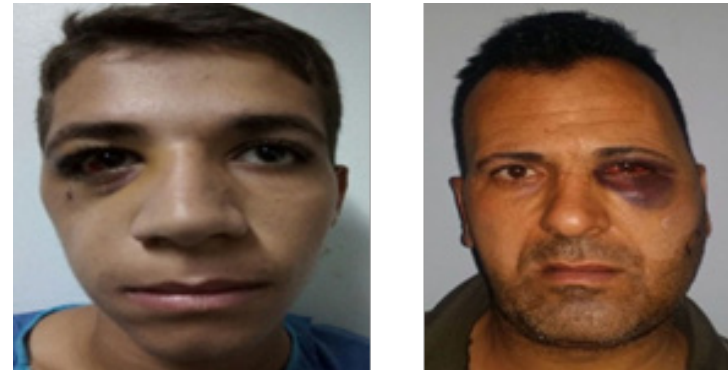

Fig.2: Preoperative photographs frontal view for manual group (a) with Rt. zmc fracture and stl group(b) with Lt. Zmc fracture.

\section{STL fabrication}

Serial computed tomographic images in multiple planes were taken. CT data presented in DICOM file (Digital Imaging and Communications in Medicine) were imported into specialized medicalsoftware program ( MIMICS ) to create 3D virtual model by Computer Aided Design (CAD). The unaffected orbit was mirrored onto the contralateral side (i.e. the traumatized orbit) (Fig.3). The virtual model data were converted to STL format to create a solid physical orbital model using a $3 \mathrm{D}$ printer and computer-aided manufacturing machines. Titanium mesh was adapted and contoured on the model preoperatively and sent for sterilization (fig.4).
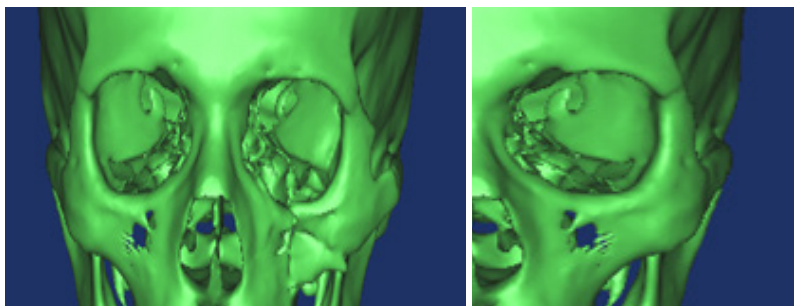

Fig.3: (a) preoperative 3D of Rt intact orbit and Lt fractured one , (b) Lt orbit after mirroring by Rt one

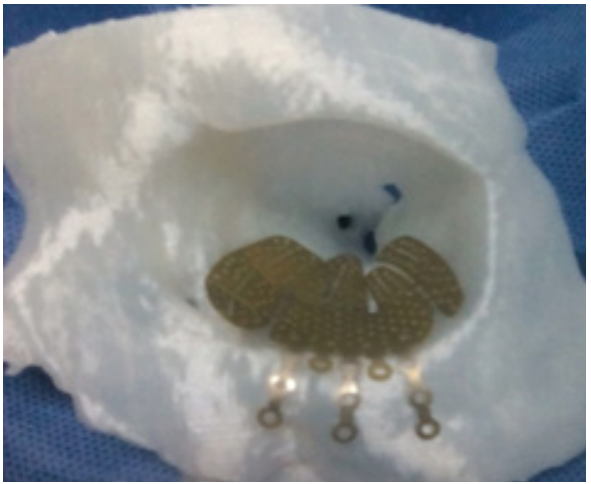

Fig.4: preoperative adaptation of titanium mesh on stl model

\section{Orbital volume measurement}

CT scans with high resolution osseous window level setting were obtained. The images were transferred to a 3-D reconstruction software program. The bony orbit on each scan was measured with the cursor to trace the orbital wall on the screen. On the axial CT scans, the anterior orbital boundary was defined by a straight line connecting the medial and the lateral orbital rims, and the regions lacking a bony boundary (ie, inferior orbital fissure, superior orbital fissure, optic canal, and lacrimal fossa) were traced with a straight line. After tracing with the cursor, a filling option can be used to inject the orbit with a digital dye6. The areas of these outlines were measured for each scan and summed for orbital volume calculation. Coronal CT scans were used to demarcate the extent of the orbital volume ${ }^{[7]}$. (Fig.5).
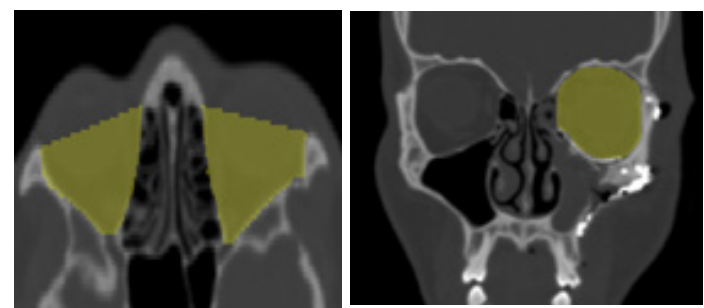

Fig.5 : Measurement of orbital volume on CT scan with tracing technique and dye filling. (a) Preoperative axial view for Rt intact orbit and Lt fractured one, (b)Postoperative coronal view for repaired Lt orbit.

\section{Surgical technique}

Under general anesthesia, the orbital floor was exposed using transcutaneous approach. The periosteal incision was made and subperiosteal dissection was carried out. The orbital soft tissue was lifted out of the fractured site, repositioned in the orbit, and the boundaries of the orbital defect were identified. The entire wall defect exposed backward up to the posterior border of defect. The orbital rim was reduced and fixed with a $2.0-\mathrm{mm}$ plate. For stl group, insertion and positioning of the pre-adapted titanium mesh was performed with reference to the landmarks of stl model with no or minimal adjustment. For manual group, bending and cutting of the mesh was performed intraoperatively with multiple trials. One or two titanium screws 
were used for mesh fixation (Fig.6). A forced duction test was done to exclude soft tissue entrapment. Intravenous antibiotics prophylaxis were used, and the patient was discharged home 2 or 3 days after surgery. CT scans were requested postoperatively.
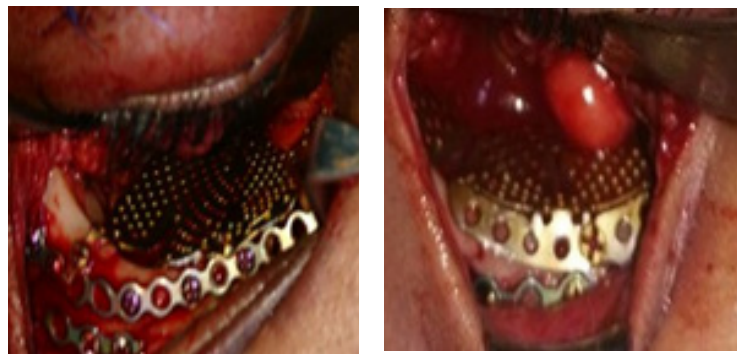

Fig.6: intraoperative photographs showing titanium mesh in place for manual group (a) and STL group (b)

\section{Clinical results}

All patients underwent surgical procedure with clinically acceptable outcome. Open reduction and internal fixation done within 2 weeks. Diplopia was found in one patients of stl group preoperatively and it did not resolve postoperatively. Retinal detachment was found in one patient of manual group. One patient in stl group suffered enophthalmos, left central scotoma, and vertical dystopia pre-operatively and there was improvement postoperatively in the dystopia and enophthalmos. Infraorbital hypoesthesia was documented in four patients in each group preoperatively and resolved postoperatively. Ectropion was found postoperatively in two patients of manual group and one patient of stl group. (Fig.7).
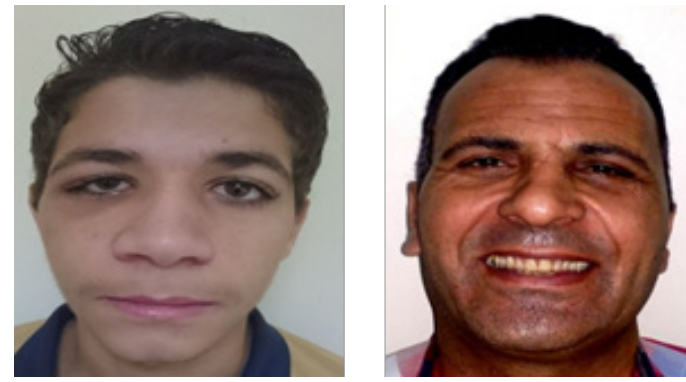

Fig.7: Postoperative photographs: frontal view for manual group (a) with repaired Rt orbit and stl group (b) with repaired Lt.

\section{Radiographic results}

Post-operative CT scans revealed acceptable reduction and fixation of fractured orbit in the both groups. Orbital volume measurements were recorded pre and postoperatively for both groups. The mean (SD) of intact contralateral orbital volumes was $28.2 \pm(2.3) \mathrm{mL}$ and $26.3 \pm(4.3) \mathrm{mL}$, for stl and manual group respectively. The average preoperative orbital volumes on the fractured side was $32.8 \pm$ (3.6) $\mathrm{mL}$ and $28.4 \pm$ (5.4) $\mathrm{mL}$, for stl and manual group respectively, while the average postoperative orbital volumes was27.3 \pm (1.9) $\mathrm{mL}$ and $26.8 \pm$ (4.8) $\mathrm{mL}$ for stl and manual group respectively (Table 1). There was statistically significant difference between pre and postoperative orbital volumes in both groups however, there was no statistical difference in percentage of correction between stl group and manual group. (Table 2) (Figs 8,9).

Table 1: mean orbital volume

\begin{tabular}{cccc}
\hline \multicolumn{4}{c}{ Bony Orbital Volume, Mean (SD), mL } \\
\hline & \multicolumn{2}{c}{ Intact orbit } & \multicolumn{2}{c}{ Affected orbit } \\
\hline & & repaired & fractured \\
STL group & $28.2(2.3)$ & $32.8(3.6)$ & $27.3(1.9)$ \\
Manual group & $26.3(4.3)$ & $28.4(5.4)$ & $26.8(4.8)$ \\
\hline
\end{tabular}

Table 2: percentage of correction

\begin{tabular}{cc}
\hline \multicolumn{2}{c}{ Percentage of correction } \\
\hline Stl group & Manual group \\
$102.8(4.4)$ & $98.4(2.6)$ \\
\hline
\end{tabular}
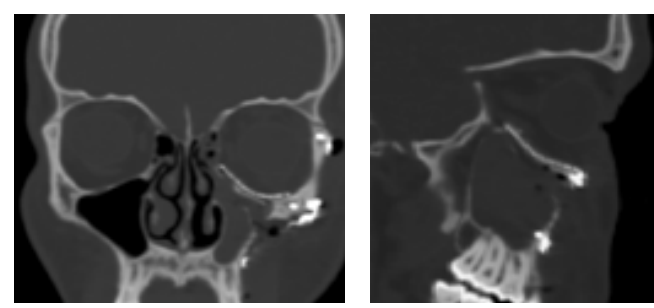

Fig.8: Postoperative CT showing orbital mesh in place for stl group. cornal view (a) and sagital view (b).
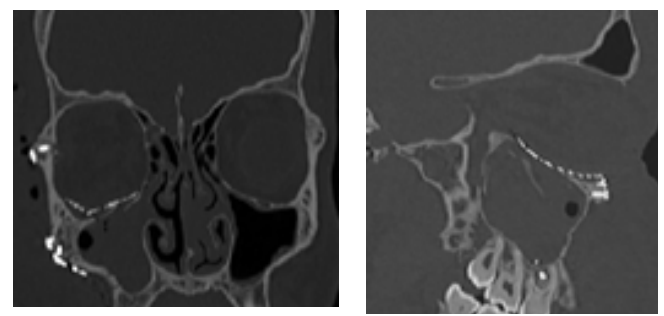

Fig.9: Postoperative CT showing orbital mesh in place for manual group. cornal view (a), sagital view (b).

\section{DISCUSSION}

Symmetrical orbital reconstruction is very important for both functional and aesthetic reasons1. Restoration of orbital anatomy is not a simple task due to complexity of orbital floor topography, which necessities accurate adaptation and contouring of the grafting material. Titanium mesh is most commonly used material for restoration of floor continuity. However, mesh adaptation is a critical step in orbital volume restoration. Enophthalmos, diplopia, muscle entrapment, and visual acuity disturbance are severe complications in posttraumatic orbital deformities due to orbital enlargement and improper correction of orbital anatomy ${ }^{[8]}$.

There are many techniques to reconstruct the bony orbital defects in the literature. Different kinds of autologous and/or alloplastic materials are inserted into the orbit. ${ }^{[9]}$. Evaluating the degree of augmentation is usually an intraoperative decision, and depends on the surgeon's 
experience ${ }^{[10]}$. After the operation, a volume excess persists in some patients and leading to an enophthalmos, especially if the deep orbital cone area was affected or scarring occurred10. The aim of restoring the normal contours of the orbit while avoiding postoperative complications remains difficult to achieve ${ }^{[11]}$. The exact reconstruction of the complex 3D anatomy of the orbit with an individually S-shaped rise of the floor, and a retro-bulbar bulge formed by the posterior aspect of the medial wall and the floor remains a challenge for the reconstructive surgeons and takes multiple trials to achieve this goal ${ }^{[12]}$.

Titanium mesh is most commonly used for orbital floor reconstruction because it is stable, less expensive, readily available, biologically compatible, malleable, and can be sterilized, however, the accurate adaptation of the titanium mesh intra-operatively is not easy process especially in comminuted cases with increased operative time and complications $^{[5]}$.

Within recent years, advances in imaging techniques for diagnostics and associated technologies (i.e., stereo lithographic model) have resulted in improvement in the preoperative planning of craniomaxillofacial surgeons. It is possible to facilitate the operative procedures in complicated cases by using computer-assisted preoperative planning and pre-adapting titanium mesh for orbital floor reconstruction combined with stl models to give a more accurate treatment and save time ${ }^{[1]}$.

Orbital volume measurement after maxillofacial trauma provides a significant data for estimating the severity of the injury and prevention of possible complications resulted by enophthalmos ${ }^{[13]}$. In1985, Forbes et al. described a method for calculating the orbital volume using CT images ${ }^{[14]}$. Since then, many methods have been described to calculate the orbital volume by the development of layered imaging techniques, MRI and $\mathrm{CT}^{[15]}$. Slice-by-slice manual segmentation method needs CTscans only to measure the volume, but it is an observer dependent process ${ }^{[16]}$.

A Cadaver-based study by Strong et al . revealed that patient-specific implants adapted on STL model gave the best reconstructive contour but they were expensive and time-consuming to fabricate while freehand bent implants were less expensive and less contour accuracy ${ }^{[8]}$.

Christopher et al. found that direct intraoperative trimming and adaptation may take long operative time depending on the extension of the defect and experience of the surgeon when compared with stl models while preoperative rapid prototyping reduces the intraoperative time, risk of orbital mesh malposition, poor anatomical contour, and trauma to soft tissue because of multiple insertions during trimming and adaptation of the titanium $\operatorname{mesh}^{[17]}$

Ye et al. studied 16 coronal CT scans of pure orbital floor fractures and reported that the mean (SD) orbital volume of the unaffected orbit was $23.94 \pm(3.47) \mathrm{cm}^{3}$ and the orbital volume of the fractured orbit was $28.16 \pm(4.32)$ $\mathrm{c} \mathrm{m}^{3}$ versus $24.08 \pm(3.22) \mathrm{cm}^{3}$ for the repaired orbit ${ }^{[18]}$ Their results are similar to the results obtained in this study.

Our results are similar to the results of Kwon et al. and revealed that it is very hard to measure the exact bony orbital volume, and to achieve it even after orbital reconstruction ${ }^{[7]}$. It was noted that the overcorrection in stl group is an advantage to compensate and overcome any degree of late enophthalmos which may occur in the future as a result of orbiral fat atrophy.

\section{Study limitations}

There are many limitations to our study. The first is a small sample size. A larger sample size might reveal a statistically significant difference between the 2 treatment groups. Second is the use of certain type of titanium mesh. Use of different types titanium mesh can allow wide comparison. Third is fabrication of stl model from PLA. Use of more accurate printing techniques and different materials for stl fabrication might reveal significant results. In the future, the use of intraoperative imaging methods ${ }^{[19]}$ and navigation ${ }^{[20]}$ may further promote the results and the understanding of this problem.

\section{CONCLUSIONS}

Although there was no significant difference in orbital volume between the two methods of treatment, application of pre-adapted titanium mesh by the aid of stl model provides a successful tool to correct increased orbital volume secondary to orbital floor fracture with less effort and operative time. Comminuted and multiple fractures cases are the best candidate for such technique to ensure a good clinical outcome and thereby decrease the incidence of complications.

\section{REFERENCES}

1. Metzger MC, Schön R, Schulze D, Carvalho C, Gutwald R, Schmelzeisen R. Individual preformed titanium meshes for orbital fractures. 2006;442-7.

2. Ahn HB, Ryu WY, Yoo KW, Park WC, Rho SH, Lee $\mathrm{JH}$, et al. Prediction of Enophthalmos by Computer-Based Volume Measurement of Orbital Fractures in a Korean Population. 2008;24(1):369.

3. Maglione M, Sossi D, Cecchini P, Rizzo R. Orbital Floor Reconstruction in Facial Asymmetry: A Clinical Case ClinMed. 2015;3-6.

4. Scolozzi P, Momjian A, Heuberger J. ComputerAided Volumetric Comparison of Reconstructed Orbits for Blow-Out Fractures With Nonpreformed Versus 3-Dimensionally Preformed Titanium Mesh Plates : A Preliminary Study. 2010;34(1):98-104.

5. Liss J, Stefko ST. Orbital Surgery : Sta te of the Art. Oral Maxillofac Surg Clin NA [Internet]. Elsevier Ltd; 2010;22(1):59-71. Available from: http://dx.doi.org/10.1016/j.coms.2009.11.006 
6. Falguera MI, Millan JM. Degrees of Tolerance in Post-Traumatic Orbital Volume Correction: The Role of. YJOMS [Internet]. Elsevier Inc.; 2009;67(11):2404-11. Available from:http:// dx.doi.org/10.1016/j.joms.2008.11.024

7. Measurements of Orbital Volume Change Using Computed Tomography in Isolated Orbital Blowout Fractures. 2015;11(6):2007-10.

8. Metzger MC, Zumbansen J, Wilson MD. Preformed vs Intraoperative Bending of Titanium Mesh for Orbital Reconstruction. 2013;(March).

9. Pagliaro F. Posttraumatic Enophthalmos: Etiology, Principles of Reconstruction, and Correction. 2005;351-9.

10. Koppel DA, Foy RH, Mccaul JA, Logan J, Hadley M, Ayoub A. The reliability of " Analyze "” software in measuring orbital volume utilizing CTderived data. 2003;5182:88-91.

11. Cho RI, Davies BW. Combined Orbital Floor and Medial Wall Fractures Involving the Inferomedial Strut: Repair Technique and Case Series Using Preshaped Porous Polyethylene / Titanium Implants. 2013;1(212).

12. Scho R. Individually preformed titanium mesh implants for a true-to- original repair of orbital fractures. 2006;990-5.

13. Pabqcuj Y. Measurement of Orbital Volume by a S-Dimensional Software Program: An Experimental Study. 2000;

14. Forbes G, Gehring DG, Gorman C a, Brennan MD, Jackson IT. Volume measurements of normal orbital structures by computed tomographic analysis. AJR Am J Roentgenol [Internet]. 1985;145(1):149-54. Available from: http://www. ncbi.nlm.nih.gov/pubmed/3873836

15. Mottini M, Wolf CA, Jafari SMS, Katsoulis K, Schaller B. Stereographic measurement of orbital volume, a digital reproducible evaluation method. 2017;1-5.

16. Jansen J, Schreurs R, Dubois L, Maal TJJ, Gooris PJJ, Becking AG. Orbital volume analysis : validation of a semi-automatic software segmentation method. 2015;

17. Lim CGT, Campbell FDI, Clucas DM, Mech N Rapid Prototyping Technology in Orbital Floor Reconstruction: Application in Three Patients. 2014;1(212):143-6.

18. Ye J, Kook KH, Lee SY. Evaluation of ComputerBased Volume Measurement and Porous Polyethylene Channel Implants in Reconstruction of Large Orbital Wall Fractures. 2006;M:509-13.

19. Hoelzle F, Klein M, Schwerdtner O, Lueth T, Albrecht J, Hosten N, et al. Intraoperative computed tomography with the mobile CT Tomoscan M during surgical treatment of orbital fractures. Int J Oral Maxillofac Surg [Internet]. 2001;30(1):2631. Available from: http://linkinghub.elsevier.com/ retrieve/pii/S0901502700900146

20. Hohlweg-Majert B, Schön R, Schmelzeisen R, Gellrich N-C, Schramm A. Navigational maxillofacial surgery using virtual models. World J Surg [Internet]. 2005;29(12):1530-8. Available from: http://www.ncbi.nlm.nih.gov/ pubmed/16311844 